\title{
AUTOIMMUNE LYMPHOPRÉLATIF SYNDROME (ALPS) : A CASE WITHOUT CYTOPENIA
}

N. Djobbi, Besbes , H. Kammoun, S. Bouguerra, O. Maatouk, D. Braham, C. Chouchane, S.Chouchane faculty of medecine of monastir-universiy of monastir, peadiatric, - Monastir, Tunisia.

\section{Background and Aims :}

Autoimmune lymphoprélatif syndrome (ALPS) is a rare inherited disorder, charectirized clinically by lymphadenopathy, hepatosplenomegaly and autoimune maniefstation. Severe immunological cytopenia is usually found in ALPS syndrome.

\section{Case report :}

A 12 years-old boy was admitted for polyadénopathy that appeared three years ago.

There was no significant past medical history .

The physical examination noted : apyrexia, hepatomegaly at $14 \mathrm{~cm}$, splenomegaly at $12 \mathrm{~cm}$, multiple cervical lymphadenopathies jugulo carotid, firm and mobile without local inflammatory signs.

The complementary examinations has eliminated malignancy and common infectious causes : Blood counts was normal specially no Thrombocytopenia.

A lymph node biopsy showed chronic non specific hyperplasic adenitis , TST reaction and Serologies (HIV,CMV,EBV,Parvovirus B19,Toxoplasma) were negative.

Also, the autoimmunity assement showed a positive antinuclear antibodies (anti SSA,SSB, antinucléosome and antihistone). A TAP scan showed multiple lymphadénopathy under and upper the diaphragm with an hepatosplenomegaly and without any lymphomatous transformation.

The diagnosis of ALPS syndrome was based on the following arguments:

- Chronic (> 6 months)

- Non malignant, non infectious lymphadenopathy or splenomegaly or both

- Elevated CD3+TCRa $\beta+C D 4-C D 8-D N T$ cells with normal lymphocyte counts.

\section{Discussion :}

- Autoimmune lymphoproliferative syndrome is a rare heritable disorder.

- He prevalence of ALPS is unknown. It has been characterized in more than 500 patients to date and has been reported worldwide in various ethnic groups.

- Depending on the specific genetic mutation, inheritance may be autosomal dominant or autosomal recessive.

- The disease course is also variable. Several genetic subtypes based on the causative genes and types of mutations have been proposed.

- The underlying defect in some (but not all) patients is a mutation in the tumor necrosis factor receptor superfamily 6 .

- This results in a defective Fas-mediated apoptosis and polyclonal expansion of a T-cell.

- Most cases present with lymphoproliferation (lymphadenopathy, hepatosplenomegaly) with or without manifestation of autoimmunity.

- Clinical manifestations of autoimmunity in the form of hemolytic anemia, thrombocytopenia, neutropenia, or are of variable severity but these signs are often absent at the time of diagnosis like our patient.

- Some patients may require chronic immunosuppressive therapies with sirolimus and mycophenolate mofetil.

- The prognosis for ALPS patients remains guarded.

- ALPS-FAS patients have a significantly increased risk of non-Hodgkin's and Hodgkin's lymphoma which can occur at any age and responds to conventional chemotherapy

\section{Conclusion :}

ALPS Syndrome is a rare condition that should be mentioned in case of an unexplained chronic lymphoproliferative disorder.

Regular monitoring of the blood count is necessary because cytopenia may be delayed. 